\title{
Manifold Dynamic Non-Covalent Interactions for Steering Molecular Assembly and Cyclization
}

\section{Shaotang Song}

National University of Singapore

Lulu Wang

National University of Singapore

Jie Su

National University of Singapore

Zhen Xu

Institute of Advanced Energy, Kyoto University

Chia-Hsiu Hsu

National Sun Yat-sen University

Chenqiang Hua

Zhejiang University

Pin Lyu

National University of Singapore

Jing Li

National University of Singapore

https://orcid.org/0000-0002-5627-4153

Xinnan Peng

National University of Singapore

Takahiro Kojima

Kyoto University

Shunpei Nobusue

Kyoto University

Mykola Telychko

National University of Singapore

\section{Yi Zheng}

Zhejiang University

\section{Feng-Chuan Chuang}

National Sun Yat-sen University

Hiroshi Sakaguchi

Kyoto University

\section{Ming Wah Wong}

National University of Singapore

Jiong Lu ( $\nabla$ chmluj@nus.edu.sg ) 
Department of Chemistry, National University of Singapore https://orcid.org/0000-0002-3690-8235

\section{Article}

Keywords: molecular assembly, cyclization, non-covalent interactions

Posted Date: June 18th, 2021

DOI: https://doi.org/10.21203/rs.3.rs-636761/v1

License: (c) (i) This work is licensed under a Creative Commons Attribution 4.0 International License. Read Full License 


\section{Abstract}

Deciphering rich non-covalent interactions that govern many chemical and biological processes is crucial for the design of drugs and controlling molecular assemblies and their chemical transformations. However, real-space characterization of these weak interactions in complex molecular architectures at single bond level has been a longstanding challenge. Here, we employed bond-resolved scanning probe microscopy combined with an exhaustive structural search algorithm and quantum chemistry calculations to elucidate multiple non-covalent interactions that control the cohesive molecular clustering of a well-design precursor and their chemical reactions. The presence of two flexible bromo-triphenyl moieties in precursor leads to the assembly of distinct non-planar dimer and trimer clusters by manifold non-covalent interactions, including hydrogen bonding, halogen bonding, $\mathrm{C}-\mathrm{H} \cdots \pi$ and lone pair $\cdots \pi$ interactions. The dynamic nature of weak interactions allows for transforming dimers into energetically more favourable trimers as molecular density increases. The formation of trimers also facilitates thermally-triggered intermolecular Ullman coupling reactions, while the disassembly of dimers favours intramolecular cyclization, as evidenced by bond-resolved imaging of metalorganic intermediates and final products. The richness of manifold non-covalent interactions offers unprecedented opportunities for controlling the assembly of complex molecular architectures and steering on-surface synthesis of quantum nanostructures.

\section{Introduction}

Non-covalent interactions span a wide range of binding energies, and consist of hydrogen bonding, halogen bonding, dipole-dipole interaction, various types of aromatic interactions, steric repulsion and London dispersion,[1] which govern a diversity of chemical and biochemical processes including chemical interactions within protein-drug, catalyst-substrate and molecular self-assemblies [2-4]. Understanding these non-covalent interactions at atomic bond level is therefore vital not only for identifying the driving forces that control the molecular arrangements in complex natural processes, but also for the design of drugs and new supramolecular architectures.

One peculiar feature of non-covalent interactions lies in their dynamic nature exemplified by great flexibility and reversibility, that can be readily influenced by external factors such as molecular density,[5] $\mathrm{pH}$ value,[6] temperature,[7] and light irradiation.[8] Despite considerable interest in this field, microscopic knowledge into the interplay between manifold non-covalent interactions, particularly at the single-bond level, that controls the formation of elegant supramolecular architectures remains elusive.

Common strategies for the characterization of the non-covalent interactions rely on the utilisation of the sample-averaged techniques including nuclear magnetic resonances, mass spectroscopy and X-ray diffraction (XRD). These techniques allow to gather information on the presence of certain non-covalent bonds in a molecular compound, but their spatial resolution is rather limited, thus making it challenging to visualize dynamic processes in supramolecular assemblies governed by multiple non-covalent interactions. 
Recent experimental breakthroughs in the tip-functionalised scanning probe microscopy (SPM) techniques demonstrate their remarkable capability for sub-angstrom real-space imaging of the chemical bonds and elucidation of the intermolecular interactions in self-assembled molecular architectures[9-14]. However, the majority of these studies have been focused on planar or nearly-planar molecular structures. In addition, on-surface molecular assemblies investigated to date mainly involve a limited category of non-covalent interactions, such as halogen bond $[5,15,16]$ or hydrogen bond interactions.[17] Molecularlevel insights into complex three-dimensional (3D)-like assemblies with manifold non-covalent interactions remain largely unexplored [18].

Here, we exploited a combined scanning tunnelling microscopy (STM) and non-contact atomic force microscopy (ncAFM) techniques, corroborated by an exhaustive structural search algorithm and quantum chemistry calculations to elucidate manifold non-covalent interactions in the 3D molecular clusters. These clusters are self-assembled by a non-planar precursor molecule with two flexible bromo-triphenyl moieties adapting a V-shaped conformation. Upon the deposition of this precursor on $\mathrm{Au}(111)$, it is spontaneously organised into dimers and trimers (Fig. 1a). The dominant interactions in dimers are found to be hydrogen bonding coexisted with lone pair $\cdots \pi$ interactions. In contrast, despite the presence of hydrogen bonding, the trimer is mainly driven by halogen bonding with the formation of two $\mathrm{Br}_{3}$ synthons formed by Type-II (Fig. S1) synergistic interactions at two different vertical planes. Moreover, such manifold non-covalent interactions can be exploited to tune on-surface reaction pathways, leading to different desired products, as evidenced by atomic-scale visualization of both metalorganic intermediates and final products via bond-resolved ncAFM imaging.

\section{Results And Discussion}

STM/ncAFM measurements. Precursor 1 was synthesized via Suzuki coupling of 2,2'-dibromo-5,5'-diiodo$1,1^{\prime}$-biphenyl and biphenyl boronic acid (see synthetic details in supplementary information). Singlecrystal XRD measurements of a bulk single crystal reveal the expected atomic structures of precursor 1 which is arranged in a 3D V-shaped conformation with two triphenyl branches intersected by an angle of $75^{\circ}$ and packed in the herringbone-like conformations (Fig. S2a). A detailed analysis based on real-space non-covalent interaction $(\mathrm{NCl})$ calculations reveals that herringbone crystalline packing is governed by multiple non-covalent interactions including hydrogen bonding, $\pi \cdots \pi$ stacking, and lone-pair $\cdots \pi$ interaction (Fig. S2b).

To explore their packing geometries and non-covalent interactions in molecular assemblies in twodimensional regime at single molecule level, precursor 1 was sublimated onto $\mathrm{Au}(111)$ held at $300 \mathrm{~K}$ (see methods). Subsequent STM/ncAFM measurements were carried out at $4.5 \mathrm{~K}$ with a CO-functionalized tip. Distinct from the herringbone-like packing in 3D bulk crystals, precursor 1 forms two different molecular clusters on $\mathrm{Au}(111)$. At a low coverage of $\sim 0.1 \mathrm{ML}$, X-shaped $(\sim 40 \%)$ and $\Leftrightarrow$-shaped $(\sim 60 \%)$ molecular clusters are formed and predominately located at face-centred cubic regions or elbow sites of the $\mathrm{Au}(111)$ herringbone reconstruction as evidenced in the large scale STM image (Fig. 1c and S3a). X-shaped clusters exhibit two bright dots in the center surrounded by four "legs", whilst $\Leftrightarrow$-shaped clusters show 
three bright dots in the center surrounded by six "legs" (Fig. 1c). Such unique features indicate that X-and $\Leftrightarrow$-shaped molecular clusters are likely formed by two (dimer) and three (trimer) monomers, respectively. Moreover, we found that an increase of molecular coverage to $0.3 \mathrm{ML}$ yields a predominate presence of $\Leftrightarrow$-shaped clusters (94\%) but with a low percentage of X-shaped ones ( 6\%) (Fig. 1d and S3b).

We then performed a controlled STM tip manipulation to disassemble the clusters for the confirmation of their composition (Fig. 2). Our manipulation approach relies on applying a high voltage pulse through tip apex placed in the vicinity of the clusters (Fig. 2a). Upon the disassembly of molecular clusters, we observed two (for the X-shape) and three (for the $\Leftrightarrow$ - shape) V-shaped structures which resemble the conformation of precursor 1 (Fig. $2 \mathrm{f}-\mathrm{i}$, and $2 \mathrm{~b}$-e). This further confirms that $\mathrm{X}$ - and $\Leftrightarrow$ - shaped structures indeed represent the self-assembled dimer and trimer clusters of $\mathrm{V}$-shaped monomers. We would like to note that the bright dots attached to the $\mathrm{V}$-shaped monomer are likely attributed to the dissociated $\mathrm{Br}$ atoms due to the cleavage of $\mathrm{C}-\mathrm{Br}$ bond triggered by a voltage pulse (as highlighted by yellow circles in Fig. 2b-i).[19-21] Apart from the dominant dimer and trimer clusters, we also observed a low percentage of isolated monomers in its trans V-shape $(<1 \%)$ and cis S-shape $(<1 \%)$ configuration (Fig. $1 \mathrm{~d})$. The absence of any bright dot suggests the dissociation of $\mathrm{Br}$ atoms from precursor 1 in these monomer-like structures upon its landing on the surface. These observations indicate that self-assembly of monomers into molecular clusters tends to stabilize the precursor from surface-assisted debromination, crucial for tuning their subsequent intramolecular and intermolecular transformation.[22, 23]

To get further insight into the structural information of these molecular clusters, we performed constant height ncAFM imaging with a CO functionalized tip. The frequency shift $(\Delta f)$ contrast in such an ncAFM images reflects the magnitude of short-range forces acting between CO-decorated tip and the sample in the Pauli repulsion regime. [14, 24]. The bright areas in ncAFM images (i.e. higher $\Delta f$ value) represent regions with higher height and electron density.[25] The ncAFM images of dimer (trimer) cluster (Fig. 3b and $4 \mathrm{~b}$ ) reveal four (three) dots interconnected via sharp lines to form a rhombus (triangular) shape. The surrounding "leg-like" molecular backbones are not visible due to a large tip-molecule separation. Such a unique ncAFM contrast indicates that these molecular clusters are comprised by non-planar geometries, wherein the central bright regions are higher than the periphery.

Intermolecular interactions. According to the structural information obtained by both STM and ncAFM imaging, we then carried out an exhaustive search coupled with the quasi-Newtonian algorithm $(>200$ initial atomic structures) using first-principle calculations to find the most possible structural configuration of dimer and trimers. The low-lying energy isomers were placed on $\mathrm{Au}(111)$ to further relax using VASP. As shown in Fig. $3 \mathrm{c}$ and $4 \mathrm{c}$, strong steric hindrance between the two $\mathrm{Br}$ atoms of the precursor causes one bromophenyl motif bending up with a tilting angle of $57^{\circ}$ and $40^{\circ}$ for the dimer and trimer, respectively. As a result of such a dramatic structural geometric change, one $\mathrm{Br}$ and one $\mathrm{H}$ of each bromophenyl moiety protrude up, which are readily reflected as bright dots in the ncAFM images. Indeed, the ncAFM image of the dimer (Fig. 3c) reveals four bright protrusions associated with two $\mathrm{Br}$ and two $\mathrm{H}$ atoms from two interacting monomers. In contrast, three bright dots revealed by ncAFM image of the 
trimer (Fig. 4d) are attributed to three protruding $\mathrm{Br}$ atoms. In addition, the three protruding $\mathrm{H}$ atoms in the centre are almost invisible, due to a relatively smaller bending angle $\left(40^{\circ}\right)$ of the bromophenyl motif.

In order to gain atomic-level insight into the intermolecular interactions in these intriguing molecular assemblies, we then performed atoms in molecules (AIM) and real-space non-covalent interaction ( $\mathrm{NCl}$ ) calculations to semi-quantitatively analyse various possible contribution of the intermolecular noncovalent interactions in the dimer and trimer respectively. [26] As shown in Fig. 3d, the computed numerical electron density $[\rho(r)]$ and its Laplacian $\left[\nabla^{2}(r)\right]$ at bond critical point $(B C P)$ indicate the presence of multiple non-covalent interactions, including $\mathrm{C}-\mathrm{H} \cdots \mathrm{Br}$ (denoted as $\mathrm{a}_{1}$ and $\mathrm{a}_{2}$ ) and $\mathrm{Br}$ lone pair $\cdots \pi$ (denoted as $b_{1}$ and $b_{2}$ ), featuring the interatomic distances of $d_{\mathrm{C}-\mathrm{H} \cdots \mathrm{Br}} \approx 2.9 \AA$ and $d_{\text {lone-pair } \cdots \pi} \approx$ 3.1 Å (Fig. S4 and Table S1). The Br lone pair $\cdots \pi$ bonding represents attractive interaction between a lone pair electron of $\mathrm{Br}$ and a $\pi$-system of the up-tilted bromophenyl ring.[27-29] This type of interaction was also reported to stabilize the formation of biological macromolecules,[30, 31] and binding of inhibitors in the binding pocket of biochemical receptors.[32]

In addition, $\mathrm{NCl}$ analysis highlights [1] the attractive interaction regions as marked in green disks in the areas of $\mathrm{BCP}$ (Fig. 3h and S6). Interestingly, the lower $\mathrm{Br}$ atoms pointing towards the up-tilted bromophenyl ring lead to the emergence of lone pair $\cdots \pi$ interactions, which further increase cohesive energy of the dimer.

Compared to the dimer, the trimer features a larger number (four types) of non-covalent interactions (Fig. 4). First, the three up-tilted and three lower $\mathrm{Br}$ atoms in a trimer participate in two sets of $\mathrm{Br}_{3}$ synthons with Type-II $X_{2}$ interactions $\left(a_{7}-a_{6}\right.$ in Fig. $4 d$, Fig. S5a and S5b), acting as a main stabilizing force for the trimer, as evidenced in the BCP by AIM analysis. Significantly stronger $\mathrm{Br} \cdots \mathrm{Br}$ halogen bonds (a1-a3, $3.51 \AA$ ) are observed for the interactions of the three up-tilted $\mathrm{Br}$ atoms (Fig. S5). Electrostatic potential maps also reveal that the electropositive $\sigma$-hole pointing to the electronegative belt of the $\mathrm{Br}$ atoms in both of the $\mathrm{Br}_{3}$ synthons (Fig. $4 \mathrm{f}, \mathrm{g}$ ).

Moreover, the AIM analysis also identifies weak hydrogen bonding $\left(\mathrm{C}-\mathrm{H} \cdots \mathrm{Br}, b_{7}-b_{3}\right)$ between the up-tilted $\mathrm{Br}$ and $\mathrm{H}$ atoms from the adjacent monomers at a separation distance of $\sim 3.4 \AA$ (Fig. $4 \mathrm{~d}$ and Fig. S5c). In addition, up-tilted benzene rings are nearly perpendicular to their neighbouring bromophenyl motifs in a close proximity $(3.0 \AA)$, which leads to the formation of strong $\mathrm{C}-\mathrm{H} \cdots \pi\left(c_{7}-c_{3}\right)$ and lone pair $\cdots \pi\left(d_{1}-d_{3}\right)$ interactions (Fig. 4d, and Table S2). A combination of these attractive non-covalent interactions is further represented by green discs in the $\mathrm{NCl}$ plot (Fig. $4 \mathrm{i}$ and $\mathrm{S} 7$ ).

To gain a more quantitative understanding of these non-covalent interactions, we set out to investigate the attractive energies of these clusters by employing symmetry-adapted perturbation theory (SAPT) calculation.[33,34] The dimer and trimer reveal a total attractive stabilization energy of $30.41 \mathrm{~kJ} / \mathrm{mol}$ and $55.94 \mathrm{~kJ} / \mathrm{mol}$, respectively (Table S3). Thus, a higher ratio of the trimer than dimer is attributed not only to a higher on-surface coverage of 1 , but also to a larger energetic gain in the formation of trimers arising from a larger number of manifold non-covalent interactions. Notably, our DFT calculations reveal a rather 
negligible charge transfer between molecular precursors and $\mathrm{Au}(111)$, suggesting limited influence of molecule-substrate interactions on the non-covalent interactions (Fig. S8).

\section{On-surface transformation of precursor 1.}

The pre-arrangement of molecular precursors in the self-assembled structures can dramatically impact on-surface reaction pathways triggered by thermal annealing [35]. Here, the dimers with a relatively low stabilization energy may tend to dissociate, which thus favours intramolecular cyclization and suppresses intermolecular cyclization. In contrast, the trimers with a high stabilization energy are expected to facilitate an intermolecular coupling reaction of the debrominated biradicals.

To explore such an effect, we conducted a mild thermal annealing of the samples decorated with dimer and trimer clusters with different ratios at $400 \mathrm{~K}$. After the thermal activation of the sample, both $\mathrm{V}$ - and X-shaped products can be obtained (Fig. 5b). A statistical analysis of 3235 product molecules (at a precursor coverage of $0.35 \mathrm{ML}$ ) reveals that the yield of $91.7 \%$ and $8.3 \%$ for products 2 and $\mathbf{4}$, respectively. The ratio of these products can be tuned by controlling the precursor coverage (Fig. $5 \mathrm{~g}-\mathrm{h}$ ).

Bond-resolved ncAFM image reveals a symmetric backbone structure of product 2, consisting of a biphenylene core connected with two biphenyl branches on both sides. Two of the inner benzene rings of the biphenyl motifs are tilted due to a relief of steric hinderance, consistent with DFT calculation (Fig. S10). Magnified view of the biphenylene core unveils that the central four-membered ring is slightly brighter than the side six-membered rings (Fig. S11b). This could probably originate from the cyclobutadiene anti-aromaticity or tilting effect of the CO tip over a small size of the ring. [43] As shown in Fig. S11d, there are three possible contributing resonance structures including one cyclobutadiene and two radialene forms of the biphenylene core. Alternating single and double bonds give rise to the antiaromatic cyclobutadiene form, while four-single-bonds leading to the radialene counterpart. A detailed bond order analysis of the biphenylene motif (Fig. S11c) reveal that the four bonds have nearly the same bond length, which suggests the radialene form dominates the resonance structures.[36]

Magnified view of the X-shaped products resolves two distinct structures with a notably different contrast at the centre (denoted as products $\mathbf{3}$ and 4) (Fig. S12). The ncAFM measurements also captured their structural difference, namely, product $\mathbf{3}$ with a dark contrast at the centre and product $\mathbf{4}$ adopting a planar geometry formed by a fused hexagonal ring. Magnified ncAFM view of the central region of product 3 shows a bright dot corresponding to an Au adatom (Fig. 5d), coordinated with the four dibrominated carbon atoms. The dark ncAFM contrast is attributed to the four benzene rings that bent downwards to the $\mathrm{Au}(111)$, consistent with previous reports of organometallic compound.[36] On the other hand, product 4 shows a dibenzo[e,Ipyrene core (Fig. 5e). These results allow us to deduce a possible reaction mechanism of the Ullman coupling process from two precursors 1 (via debromination to the biradical 1') to product 4 , through the formation of metalorganic intermediate 3 (Fig. 5a).[37, 38] This intermediate provides mechanistic insight into aryl-aryl bond formation via metal-catalysed Ullmann reaction, which may serve as a new path for synthesizing polycyclic aromatic hydrocarbons. 
Competition between thermal diffusion and intermolecular reactions during thermal annealing of the selfassembled structures plays an important role in the on-surface synthesis.[39] Random diffusion of the precursor molecules with multiple reaction pathways usually leads to the yield of undesired by-products, such as uncontrolled graphene nanoribbon junctions,[40] and unexpected molecular oligomers,[41] which thus severely limits the yield of desired products. To this end, taking advantage of non-covalent interactions between the precursor reactants may provide a promising approach for steering the reaction pathways towards the desired products. Indeed, thermal diffusion of the precursors causes the disassembly of dimers at a low coverage, which facilitates intramolecular cyclization reaction leading to the biphenylene derivative 2. At an increased molecular coverage, the trimer with a larger cohesive energy dominates the assemblies. However, trimerization reaction to a highly strained product $\mathbf{5}$ is prohibited due to a large steric hindrance. As a result, intermolecular Ullman coupling reaction occurs between two of the three strongly bonded monomer, while the third monomer undergoes the intramolecular reaction (Fig. S13).

It is important to note that Cu-catalysed dimerization of precursor 1 derivative (two tert-butyl groups installed at the 4,4'-sites) in solution yields a three-dimensional Greek cross dodecaphenylene with a tetra-o-phenylene core.[42] In a stark contrast to the planar dibenzo[e,/pyrene core on the Au(111) surface, our results demonstrated that the surface-assisted reactions in two-dimensional limit can be steered by tuning multiple intermolecular non-covalent interactions, and thus proceed under distinct pathways towards the formation of the different products.

\section{Conclusion}

Real-space characterization of dynamic non-covalent interactions in molecular assemblies at atomic bond level, combined with quantum chemistry simulations allows to decode the role of multiple weak interactions on the molecular assemblies and their on-surface chemical reactions. The dynamic nature of these weak interactions allows for transformation of the arrangement of monomers in the assembled clusters as molecular density increases, which alters reaction pathways in the subsequent on-surface synthesis of cyclized products. Our findings highlight a vital route for controlling on-surface supramolecular assemblies and steering their chemical transformations through manipulation of manifold dynamic non-covalent interactions.

\section{Methods:}

Synthesis and characterization of organic precursor. Precursor 1 was synthesized according to the procedures illustrated in Scheme S2. Details of the synthesis and spectroscopic characterizations of the precursor are presented in the Supplementary Information.

Sample preparation and STM/ncAFM measurements. The STM and ncAFM measurements were performed using Scienta Omicron LT-SPM system operated at $4.5 \mathrm{~K}$ (base pressure, $1 \times 10^{-11} \mathrm{mbar}$ ). $\mathrm{Au}(111)$ single crystal (MaTeck $\mathrm{GmbH})$ was cleaned by multiple cycles of $\mathrm{Ar}^{+}$sputtering and annealing. 
ncAFM imaging was operated with a qPlus sensor (a resonance frequency of $f_{0}=22839.5 \mathrm{~Hz}$, and a quality factor of 23,000 ). ncAFM images were collected at a constant-height frequency modulation mode using an oscillation amplitude of $A=100 \mathrm{pm}$. We followed the standard procedure for the functionalization of STM tip with a CO molecule.[43] The CO functionalized tip was confirmed by scanning over another $\mathrm{CO}$ molecule that shows the characteristic change of the STM contrast. Molecular precursor was deposited by organic evaporator from a quartz crucible maintained at $430 \mathrm{~K}$ onto a clean $\mathrm{Au}(111)$ held at room temperature.

The STM tip manipulation was conducted through the following steps: (i) scan the area to identify the targeted clusters at constant current mode (1.4 V, $100 \mathrm{pA})$, (ii) position the tip at the target site in the vicinity of molecular clusters (iii) apply a voltage pulse of $-8 \mathrm{~V}$ or $-9 \mathrm{~V}$ with feedback loop on, (iv) rescan the same surface area.

Density Functional Theory calculations and structural optimization. An exhaustive search coupled with the quasi-Newtonian method was first performed for the atomic optimization using the DFT calculations. We consider the orientation and distance between the molecules and employ a combinatorial way to symmetrically generate the models. More than 200 initial models are considered for both free-standing dimer and trimer cases. The selected candidate models are placed on substrate $\mathrm{Au}(111)$ substrate for further relaxation using VASP software package V5.4.4, PBE functional[44] and a projector augmented plane wave basis set (PAW)[45] with a kinetic energy cutoff of $400 \mathrm{eV}$. The van der Waals interactions were corrected using DFT-D2 method scheme by Grimme[46]. Energies and geometry optimizations were converged to $10^{-5} \mathrm{eV}$. The $(16 \times 16)$ slab model containing one atomic layer of $\mathrm{Au}(111)$ was utilized and all the Au atoms were frozen. A vacuum of $20 \AA$ was used to separate periodic mirror images in the zdirection. The optimization was performed at a $1 \times 1 \times 1$ k-point mesh for Brillouin zone integration, which was generated by the Monkhorst-Pack method with Gamma centered.[47]

The Atoms in Molecules (AIM) and Noncovalent Interaction ( $\mathrm{NCl}$ ) analysis were performed by the Multiwfn program, Version 3.8. [48] The visualization of these analysis was using VMD[49]. The SAPT analysis was carried out at sSAPT0/jun-cc-pVDZ level of theory. [33] using PSI4 software. [45]

\section{Declarations}

Acknowledgement: J. Lu acknowledges the support from MOE grants (MOE2019-T2-2-044 and R-143000-B58-114). M. Telychko acknowledges the support from A*STAR AME YIRG grant (Project No. A20E6c0098, R-143-000-B71-305), J. Su, acknowledges the support from A*STAR AME YIRG grant (A2084c0171, R-143-000-B92-305). F-C. C. acknowledges the support from the National Center for Theoretical Sciences and the Ministry of Science and Technology of Taiwan under grant nos. MOST-1072628-M-110-001-MY3 and National Center for High-performance Computing for computer resources. Y. Zheng acknowledges the support from Zhejiang Provincial Natural Science Foundation (D19A040001). L. Wang acknowledges Applied Materials for the Ph.D. scholarship. 
Author contributions: J. L. supervised the project. S. S. and J. S. conducted the STM and ncAFM measurements. L. W. performed AIM, NCl, DFT calculations under the supervision by M. W. M. C-H.H, F-C. C., C.Q. H, and Y.Z. performed DFT calculations. S. S., Z. X., K. T. and S. N., carried out the organic synthesis under supervision of H. S.; P. L., J. L., and M. T., participated in scientific discussion. S. S. and J. L. wrote manuscript with contribution from all the authors.

\section{References}

1. Johnson, E. R. et al. Revealing noncovalent interactions. J. Am. Chem. Soc. 132, 6498-6506 (2010).

2. Cavallo, G. et al. The halogen bond. Chem. Rev. 116, 2478-2601 (2016).

3. Teyssandier, J., Mali K. S. \& De Feyter S. Halogen bonding in two-dimensional crystal engineering. ChemistryOpen 9, 225-241 (2020).

4. Shang, J. et al. Assembling molecular Sierpiński triangle fractals. Nat. Chem. 7, 389-393 (2015).

5. Lawrence, J. et al. Combining high-resolution scanning tunnelling microscopy and first-principles simulations to identify halogen bonding. Nat. Commun. 11, 1-7 (2020).

6. Le Bourvellec, C., Guyot, S. \& Renard, C. M. G. C. Non-covalent interaction between procyanidins and apple cell wall material: Part I. Effect of some environmental parameters. Biochim. Biophys. Acta, Gen. Subj. 1672, 192-202 (2004).

7. Jheng, J. F. et al. Influences of the non-covalent interaction strength on reaching high solid-state order and device performance of a low bandgap polymer with axisymmetrical structural units. Adv. Mater. 25, 2445-2451 (2013).

8. Ichimura, K. Photoalignment of liquid-crystal systems. Chem. Rev. 100, 1847-1874 (2000).

9. Temirov, R., Soubatch, S., Neucheva, O., Lassise, A. C. \& Tautz, F. S. A novel method achieving ultra-high geometrical resolution in scanning tunnelling microscopy. New J. Phys. 10, 053012 (2008).

10. Weiss, C. et al. Imaging Pauli repulsion in scanning tunneling microscopy. Phys. Rev. Lett. 105, 086103 (2010).

11. Kichin, G., Weiss, C., Wagner, C., Tautz, F. S. \& Temirov, R. Single molecule and single atom sensors for atomic resolution imaging of chemically complex surfaces. J. Am. Chem. Soc. 133, 16847-16851 (2011).

12. Mohn, F., Schuler, B., Gross, L. \& Meyer, G. Different tips for high-resolution atomic force microscopy and scanning tunneling microscopy of single molecules. Appl. Phys. Lett. 102, 073109 (2013).

13. Mönig, H. et al. Quantitative assessment of intermolecular interactions by atomic force microscopy imaging using copper oxide tips. Nat. Nanotechnol. 13, 371-375 (2018). 
14. Gross, L., Mohn, F., Moll, N., Liljeroth, P. \& Meyer, G. The chemical structure of a molecule resolved by atomic force microscopy. Science 325, 1110-1114 (2009).

15. Han, Z. et al. Imaging the halogen bond in self-assembled halogenbenzenes on silver. Science $\mathbf{3 5 8}$, 206-210 (2017).

16. Tschakert, J. et al. Surface-controlled reversal of the selectivity of halogen bonds. Nat. Commun. 11, 1-8 (2020).

17. Zhang, J. et al. Real-space identification of intermolecular bonding with atomic force microscopy. Science 342, 611-614 (2013).

18. Alldritt, B. et al. Automated structure discovery in atomic force microscopy. Sci. Adv. 6, eaay6913 (2020).

19. Pavliček, N. et al. Synthesis and characterization of triangulene. Nat. Nanotechnol. 12, 308-311 (2017).

20. Pavliček, N. et al. On-surface generation and imaging of arynes by atomic force microscopy. Nat. Chem. 7, 623-628 (2015).

21. Schuler, B. et al. Reversible Bergman cyclization by atomic manipulation. Nat. Chem. 8, 220-224 (2016).

22. Bieri, M. et al. Two-dimensional polymer formation on surfaces: insight into the roles of precursor mobility and reactivity. J. Am. Chem. Soc. 132, 16669-16676 (2010).

23. Telychko, M. et al. Strain-induced isomerization in one-dimensional metal-organic chains. Angew. Chem. Int. Ed. 131, 18764-18770 (2019).

24. Hapala, P. et al. Mechanism of high-resolution STM/AFM imaging with functionalized tips. Phys. Rev. B 90, 085421 (2014).

25. Kawai, S. et al. Direct quantitative measurement of the $\mathrm{C} \unrhd \mathrm{O} \cdots \mathrm{H}-\mathrm{C}$ bond by atomic force microscopy. Sci. Adv. 3, e1603258 (2017).

26. Bader, R. F. W. A quantum theory of molecular structure and its applications. Chem. Rev. 91, 893-928 (1991).

27. Egli, M. \& Sarkhel S. Lone pair-aromatic interactions: To stabilize or not to stabilize. Acc. Chem. Res. 40, 197-205 (2007).

28. Mooibroek, T. J., Gamez P. \& Reedijk J. Lone pair- $\pi$ interactions: a new supramolecular bond? CrystEngComm 10, 1501-1515 (2008). 
29. Gadre, S. R. \& Kumar A. Understanding lone pair- $\pi$ interactions from electrostatic viewpoint, in Noncovalent forces. 2015, Springer, Cham. p. 391-418.

30. Calabrese, J. C., Jordan, D. B., Boodhoo, A., Sariaslani, S. \& Vannelli, T. Crystal structure of phenylalanine ammonia lyase: multiple helix dipoles implicated in catalysis. Biochemistry $\mathbf{4 3}, \mathbf{1 1 4 0 3 -}$ 11416 (2004).

31. Stollar, E. J. et al. Unconventional interactions between water and heterocyclic nitrogens in protein structures. Proteins: Struct., Funct., Bioinf. 57, 1-8 (2004).

32. Li, J. et al. Strategy for discovering chemical inhibitors of human cyclophilin A: focused library design, virtual screening, chemical synthesis and bioassay. J. Comb. Chem. 8, 326-337 (2006).

33. Parker, T. M., Burns, L. A., Parrish, R. M., Ryno, A. G. \& Sherrill, C. D. Levels of symmetry adapted perturbation theory (SAPT). I. Efficiency and performance for interaction energies. J. Chem. Phys. 140, 094106 (2014).

34. Patkowski, K. Recent developments in symmetry-adapted perturbation theory. Wiley Interdiscip. Rev.: Comput. Mol. Sci. 10, e1452 (2020).

35. Zhou, X. et al. Steering surface reaction dynamics with a self-assembly strategy: Ullmann coupling on metal surfaces. Angew. Chem. Int. Ed. 129, 13032-13036 (2017).

36. Kawai, S. et al. Competing annulene and radialene structures in a single anti-aromatic molecule studied by high-resolution atomic force microscopy. ACS nano 11, 8122-8130 (2017).

37. Clair, S. \& de Oteyza, D. G. Controlling a chemical coupling reaction on a surface: tools and strategies for on-surface synthesis. Chem. Rev. 119, 4717-4776 (2019).

38. Galeotti, G. et al. An unexpected organometallic intermediate in surface-confined Ullmann coupling. Nanoscale 11, 7682-7689 (2019).

39. Otero, R. et al. Lock-and-key effect in the surface diffusion of large organic molecules probed by STM. Nat. Mater. 3, 779-782 (2004).

40. Li, J. et al. Single spin localization and manipulation in graphene open-shell nanostructures. Nat. Commun. 10, 1-7 (2019).

41. Su, J. et al. On-surface synthesis and characterization of [7]Triangulene quantum ring. Nano Lett. 21, 861-867 (2021).

42. Rajca, A., Wang, H., Bolshov, P. \& Rajca, S. A Greek cross dodecaphenylene: sparteine-mediated asymmetric synthesis of chiral $D_{2}$-symmetric $\pi$-conjugated tetra-o-phenylenes. Tetrahedron $\mathbf{5 7}, \mathbf{3 7 2 5 -}$ 3735 (2001). 
43. Bartels, L., Meyer, G. \& Rieder, K. H. Controlled vertical manipulation of single CO molecules with the scanning tunneling microscope: A route to chemical contrast. Appl. Phys. Lett. 71, 213-215 (1997).

44. Perdew, J. P., Burke, K. \& Ernzerhof, M. Generalized gradient approximation made simple. Phys. Rev. Lett. 77, 3865 (1996).

45. Blöchl, P. E. Projector augmented-wave method. Phys. Rev. B 50, 17953 (1994).

46. Grimme, S. Semiempirical GGA-type density functional constructed with a long-range dispersion correction. J. Comput. Chem. 27, 1787-1799 (2006).

47. Monkhorst, H. J. \& Pack, J. D. Special points for Brillouin-zone integrations. Phys. Rev. B 13, 5188 (1976).

48. Lu, T. \& Chen F. Multiwfn: a multifunctional wavefunction analyzer. J. Comput. Chem. 33, 580-592 (2012).

49. Humphrey, W., Dalke, A. \& Schulten, K. VMD: Visual molecular dynamics. J. Mol. Graphics 14, 33-38 (1996).

\section{Figures}


a

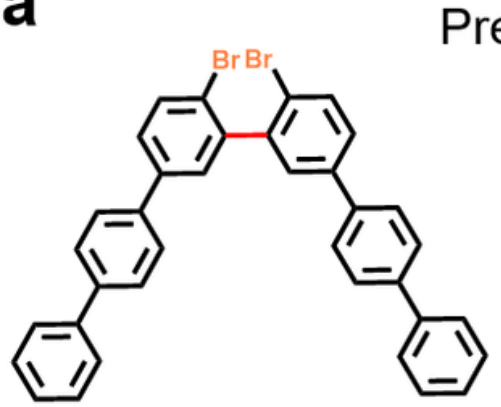

V-Conformation

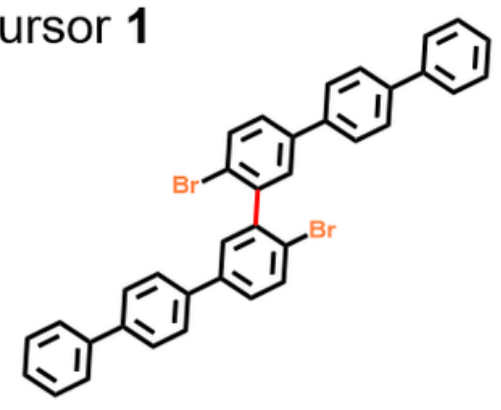

S-Conformation b

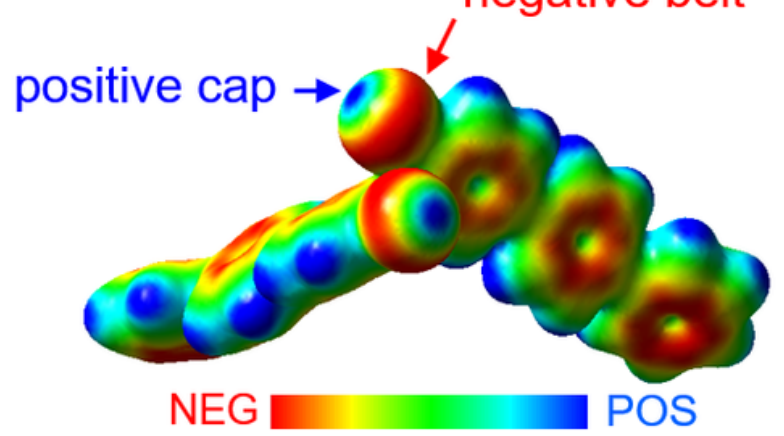

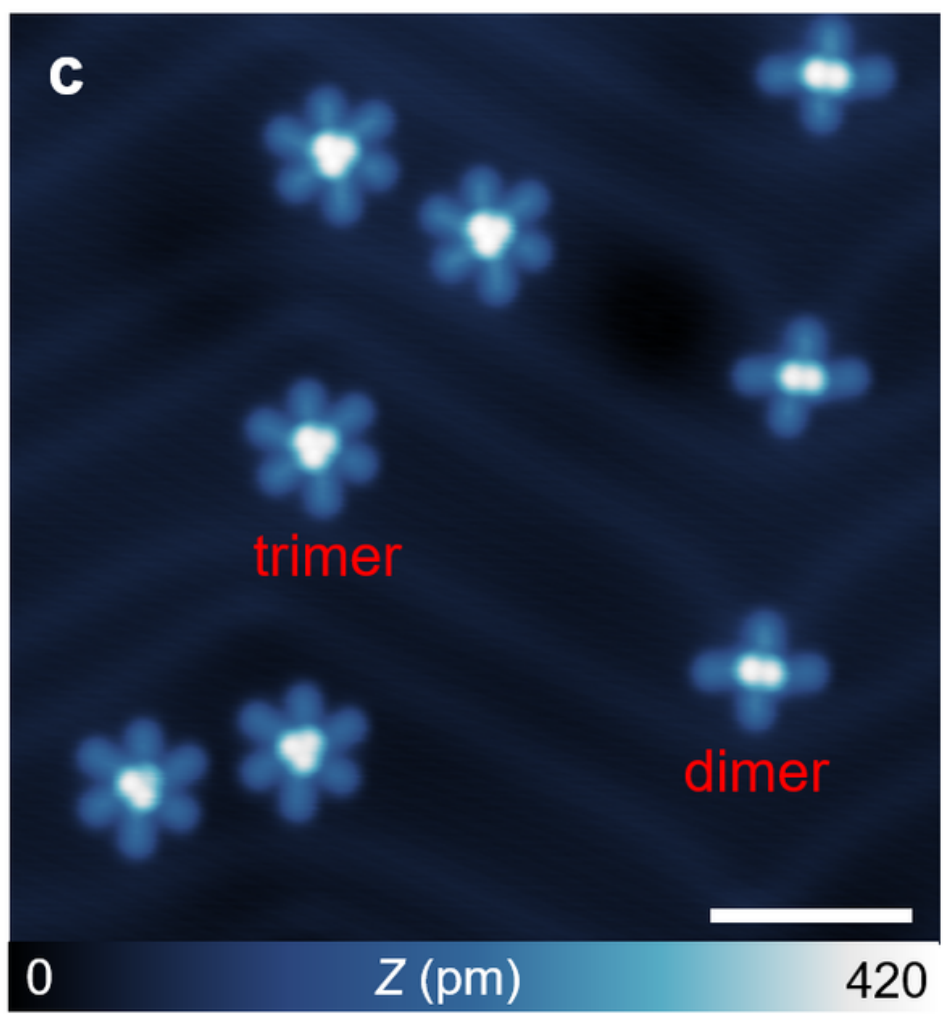

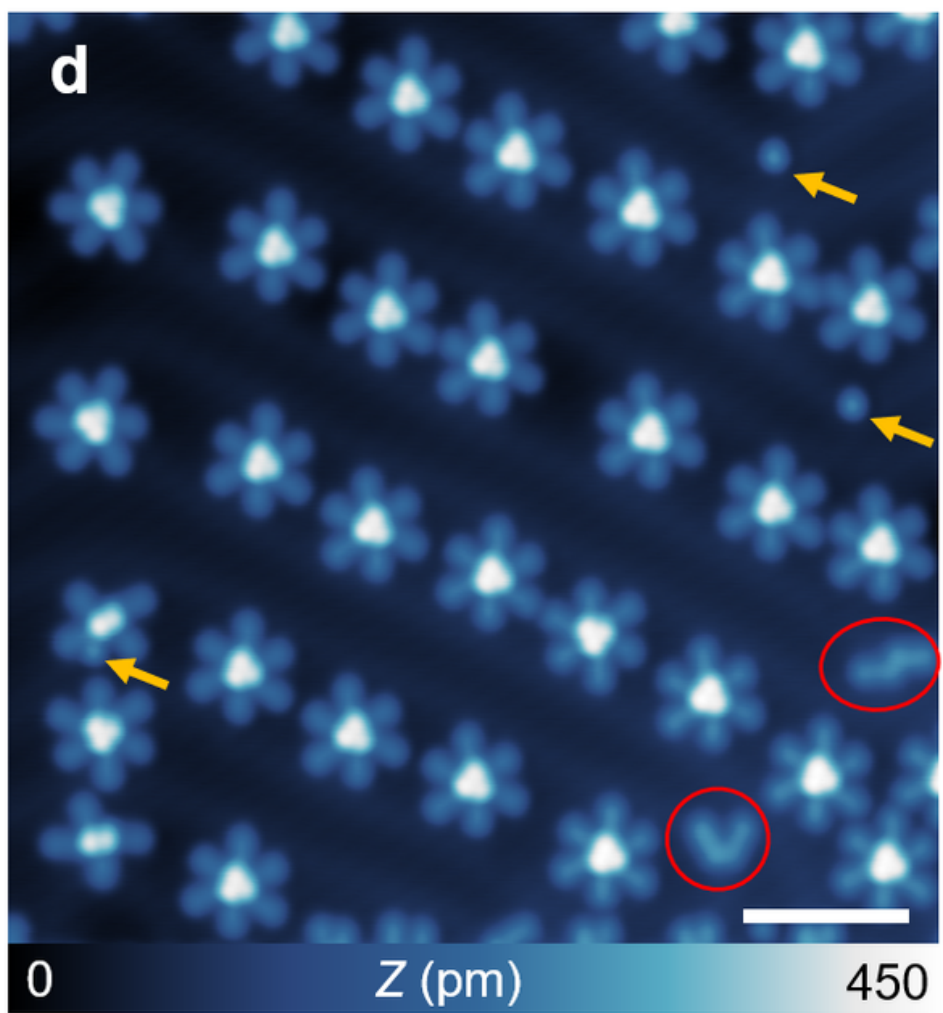

Figure 1

Self-assembly of the precursor 1 into dimer and trimer clusters on $\mathrm{Au}(111)$. a, The V-and Sconformations of the precursor upon its adsorption on $\mathrm{Au}(111)$ due to the $\mathrm{C}-\mathrm{C}$ bond rotation (highlighted in red). $b$, The electrostatic potential (ESP) map projected on an electron density isosurface (0.012 a.u.) of the electron density reveals the $\sigma$-hole (blue) and electron-rich belt (red) of the Br atoms. c-d, STM images of self-assembled molecular clusters of precursors including dimers and trimers at a low (c) and high (d) coverage, respectively. The red circles in (d) highlight the V-and S-conformations of debrominated precursor 1, respectively. The bright dots pointed by orange arrows are attributed to the disassociated $\mathrm{Br}$ atoms from the precursor monomers, which absorb over elbow sites or attached to dimer clusters. Scale bars for (c) and (d) are $5 \mathrm{~nm}$. 


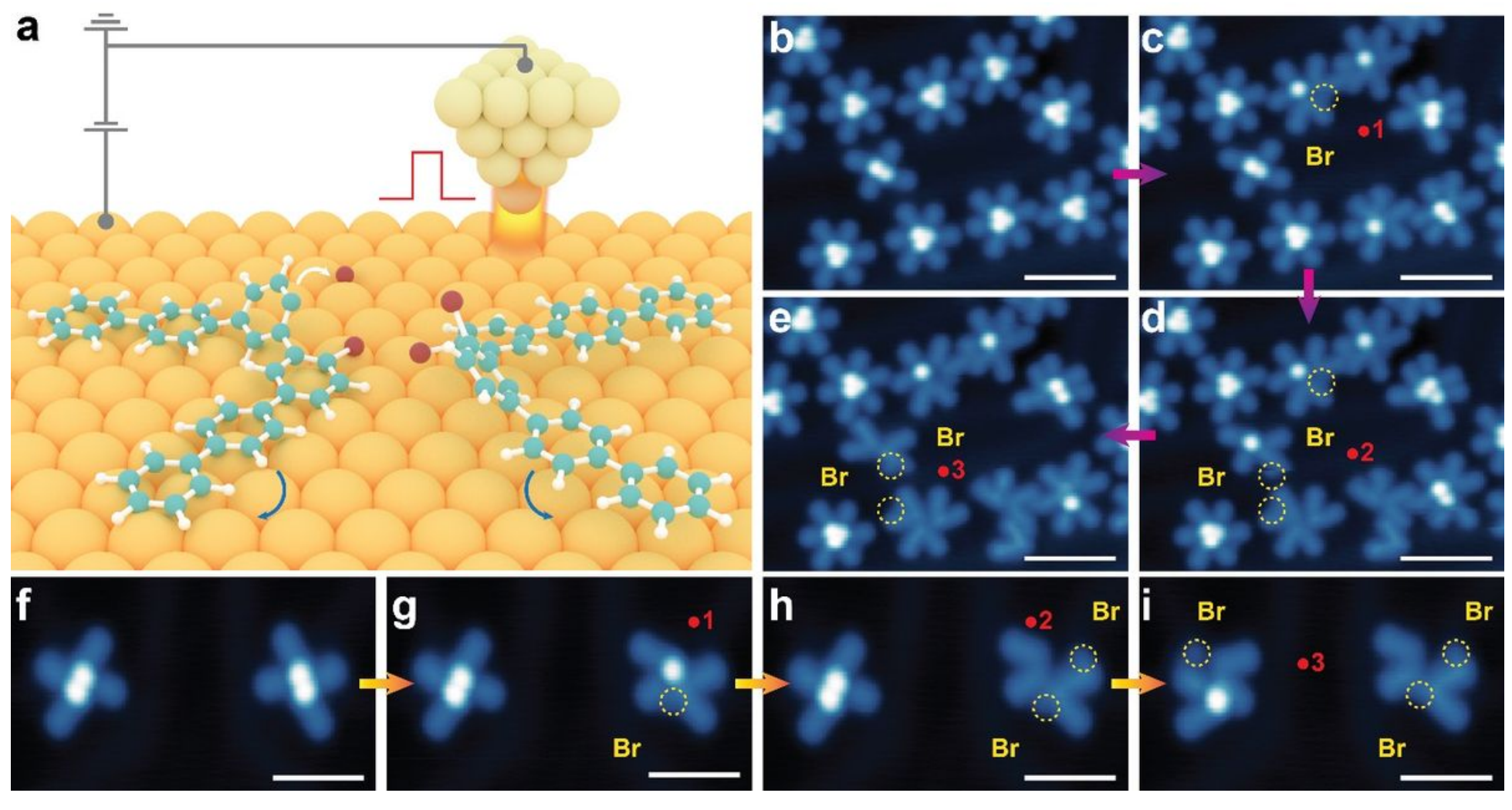

Figure 2

The disassembly of molecular clusters via STM tip manipulation. a, Schematic illustration of a voltage pulse applied in the vicinity of the cluster. It triggers the disassembly of molecular clusters via breaking the weak non-covalent interactions between precursors. $b-i$, The manipulation of the trimer $(b-e)$ and dimer (f-i) clusters. Red dots refer the positions where the voltage pulses $(-8 \mathrm{~V})$ are applied. Arabic numbers indicate the sequence for applying voltage pulses. Yellow circles highlight the $\mathrm{Br}$ atoms disassociated from the precursors by voltage pulse. Scale bars for $b-d$ are $4 \mathrm{~nm}$, and for $f-i$ are $3 \mathrm{~nm}$. 

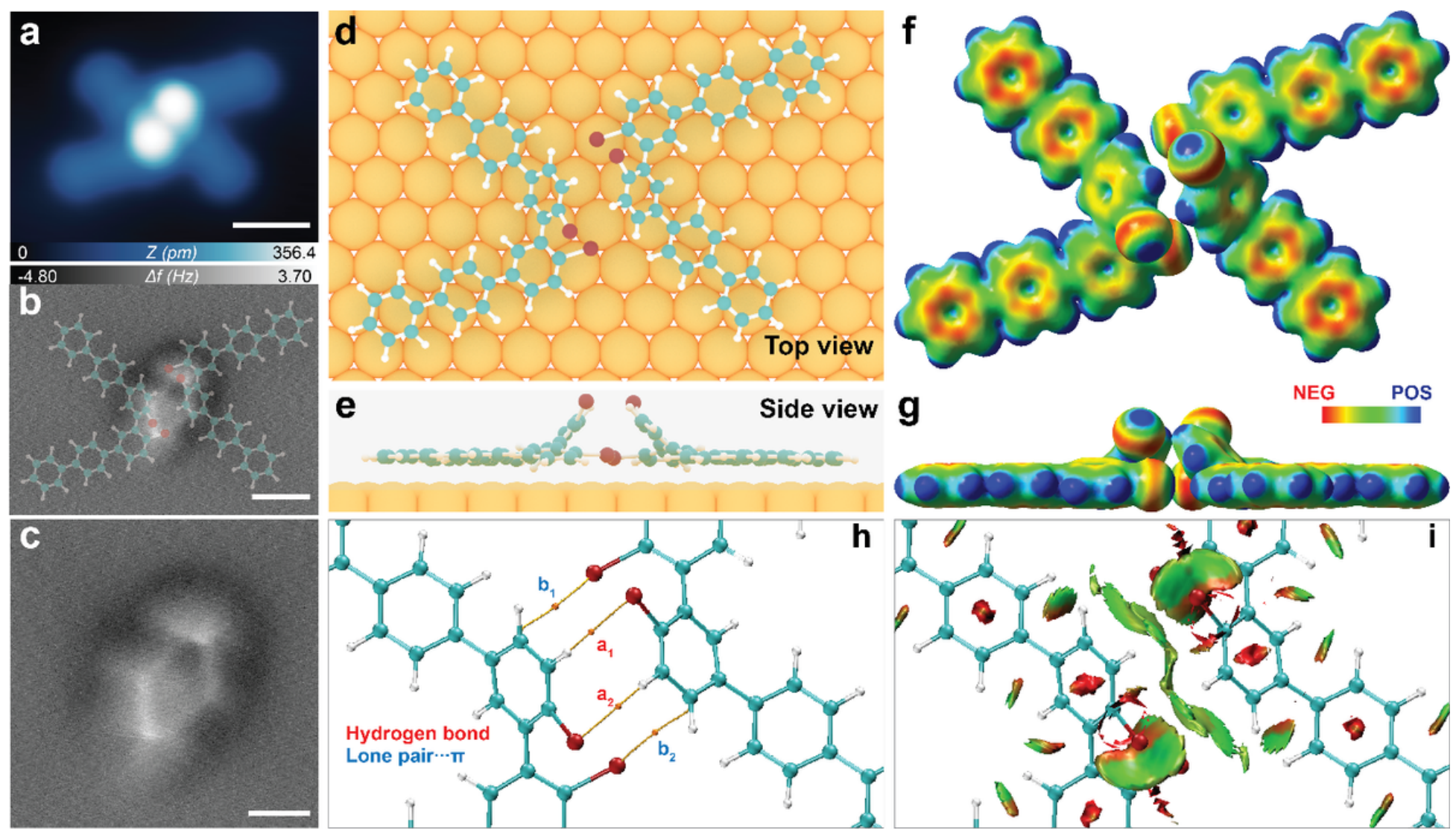

Figure 3

Characterization of multiple non-covalent interactions in dimer. a, STM image of the dimer ( $1 \mathrm{~V}, 100 \mathrm{pA})$. Scale bar is $1 \mathrm{~nm}$. b, a full-range ncAFM of the dimer with a CO functionalized tip overlaid with dimer molecular model. Scale bar is $0.5 \mathrm{~nm}$. c, zoom-in ncAFM image reveals four bright dots connected with sharp line features forming a rhombus shape. Scale bar is $0.3 \mathrm{~nm}$. d,e, Top- and side-view of the DFTmodelled dimer structure on $\mathrm{Au}(111) . \mathrm{f}, \mathrm{g}$, Top- and side-view of the electron static potential maps of the dimer. $h, B C P s$ calculation reveals the BCP of the hydrogen bonds and lone pair $\cdots \pi$ interactions revealed from AIM analysis of the dimer. i, NCl plot of the dimer showing the presence of three types of noncovalent interactions including lone pair $\cdots \pi, \pi \cdots \pi$ and $\mathrm{C}-\mathrm{H} \cdots \mathrm{Br}$ (indicated by green discs). 

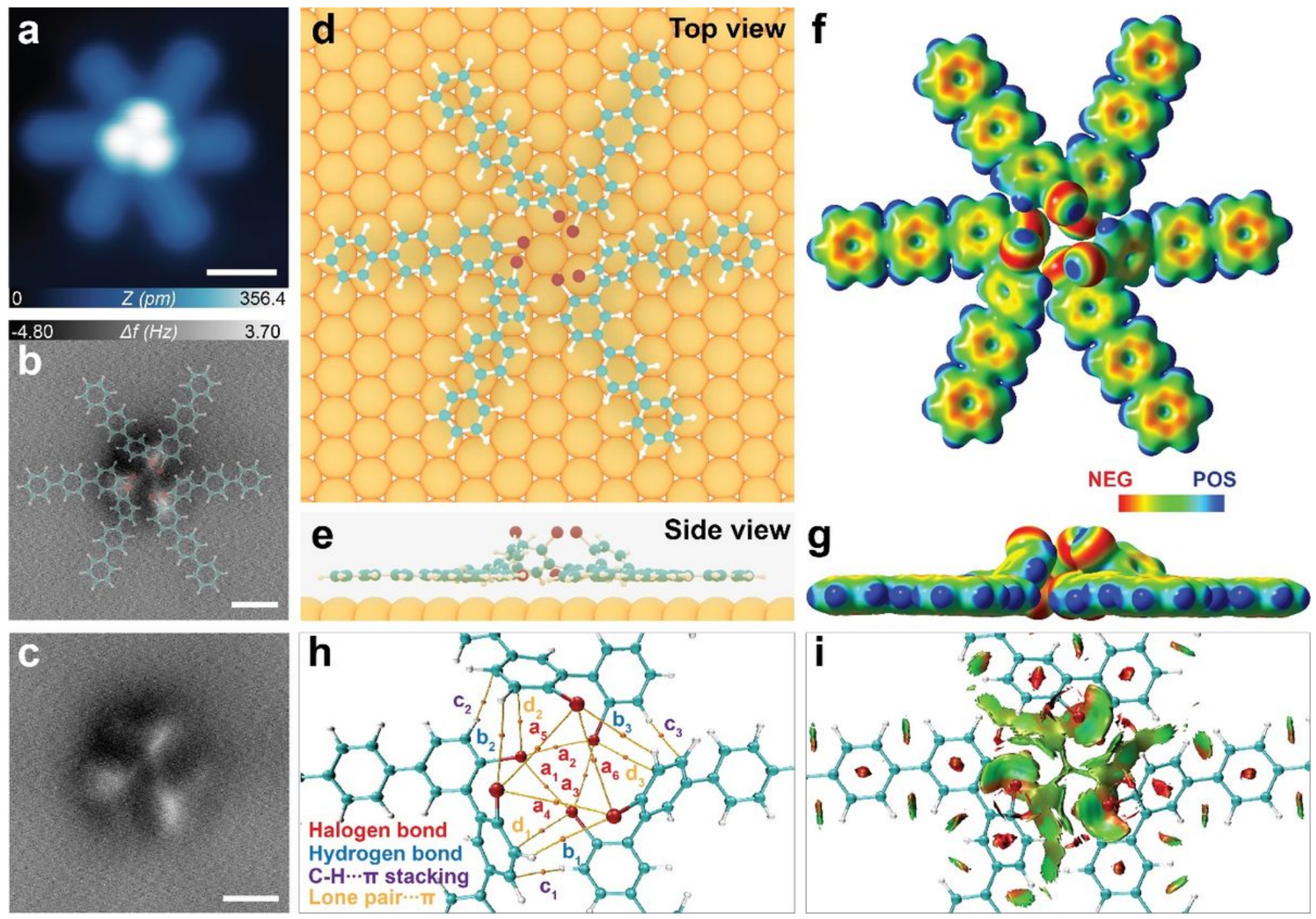

\section{Figure 4}

Characterization of multiple noncovalent interactions in trimer. a, STM image of the trimer $(1 \mathrm{~V}, 100 \mathrm{pA})$. Scale bar is $1 \mathrm{~nm}$. b, A full-range ncAFM image of the trimer with a CO functionalized tip overlaid with trimer molecular model. Scale bar is $0.5 \mathrm{~nm}$. c, Zoom-in ncAFM image shows three bright dots connected with the sharp line features forming a triangular shape. Scale bar is $0.4 \mathrm{~nm}$. d,e, Top-view and side-view of the DFT-modelled trimer structure on $\mathrm{Au}(111) ; \mathrm{f}, \mathrm{g}$, Top- and side-view of the electron static potential maps of the trimer cluster; $h, B C P s$ of the halogen bond, hydrogen bonds, $\mathrm{CH} \cdots \pi$ and lone pair $\cdots \pi$ interactions revealed from AIM analysis of the trimer; and $\mathrm{i}, \mathrm{NCl}$ plot of the trimer reveal four types of noncovalent interactions including the halogen, hydrogen, lone pair $\cdots \pi$, and $\mathrm{C}-\mathrm{H} \cdots \pi$ (indicated by green discs). 
a

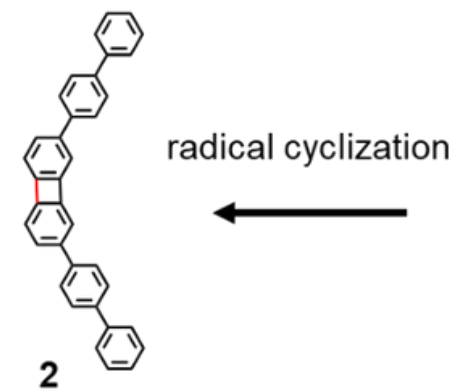

b

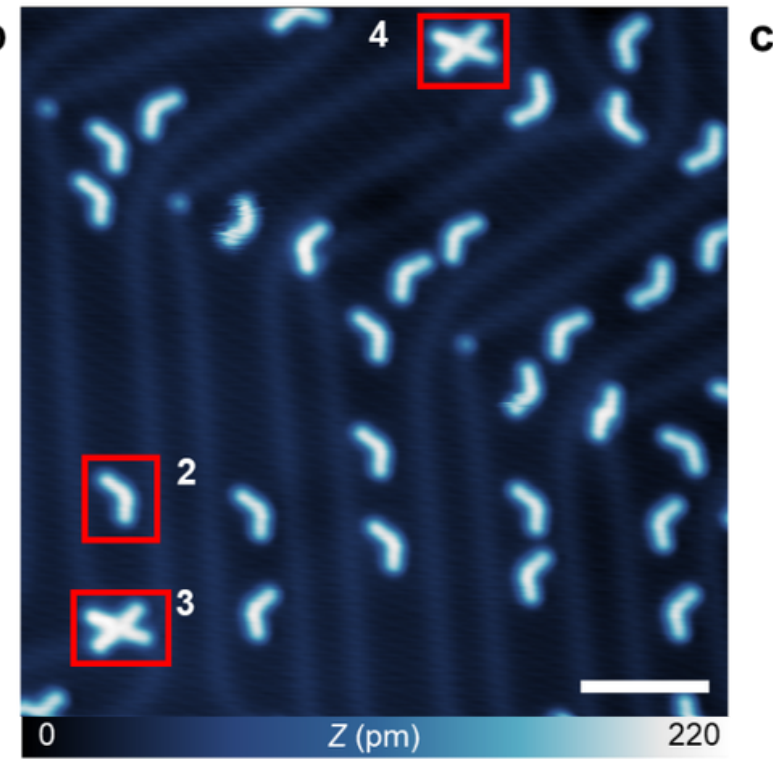

f

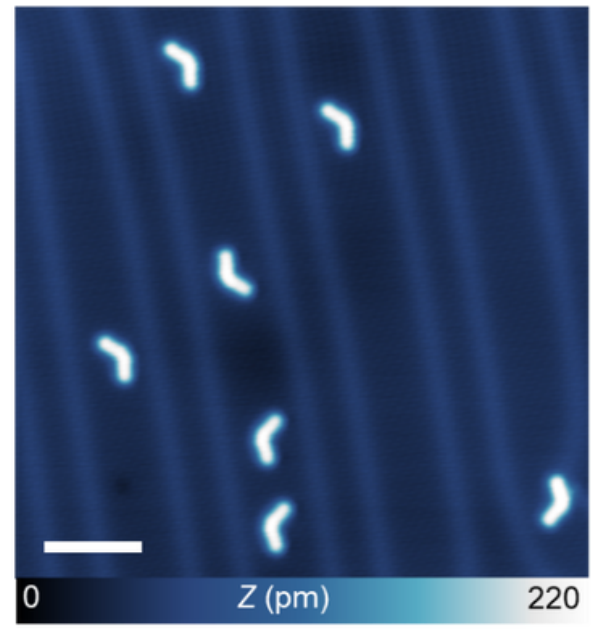

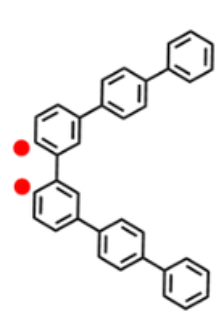

1 '

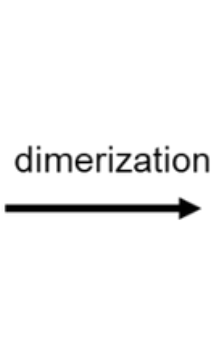

$d$
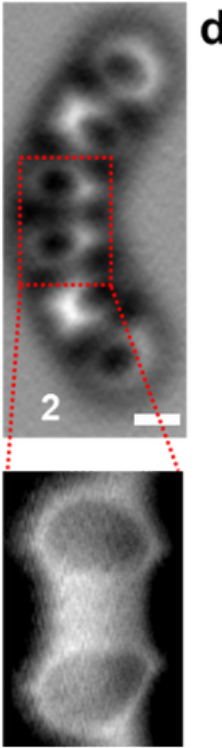

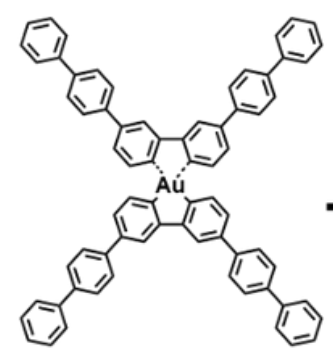

3

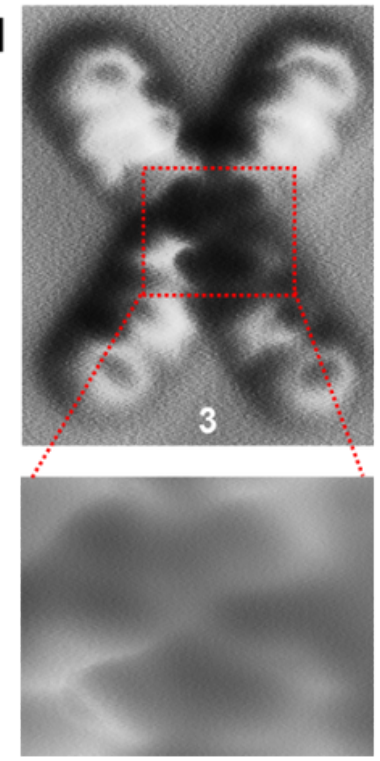

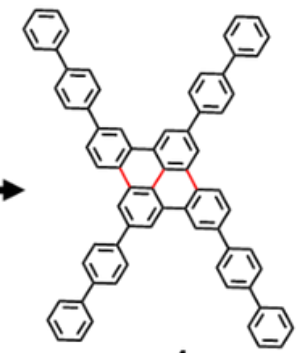

4

e
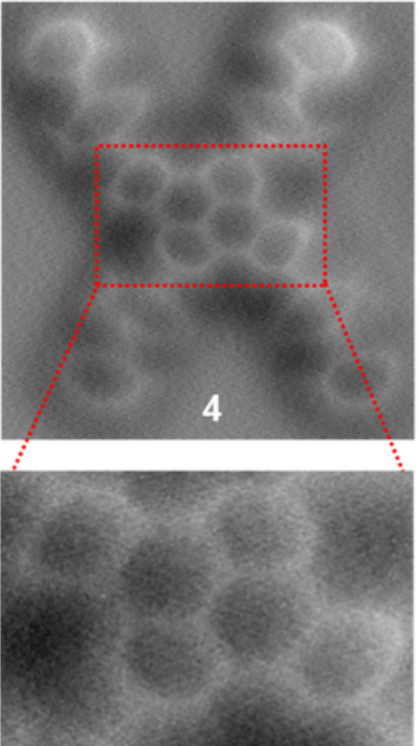

g

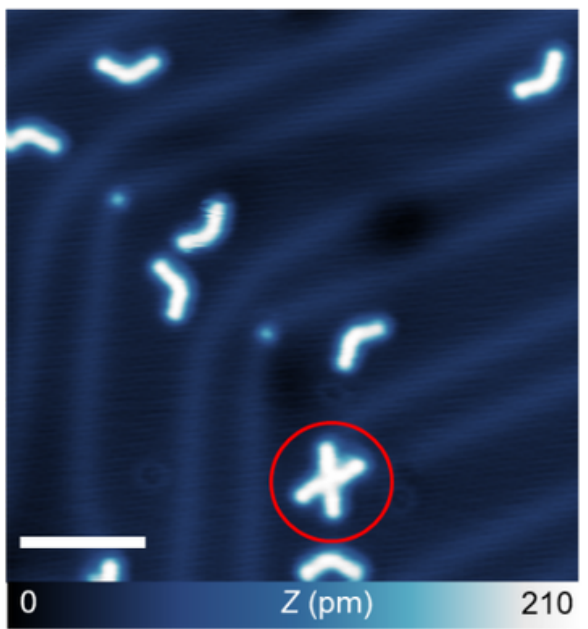

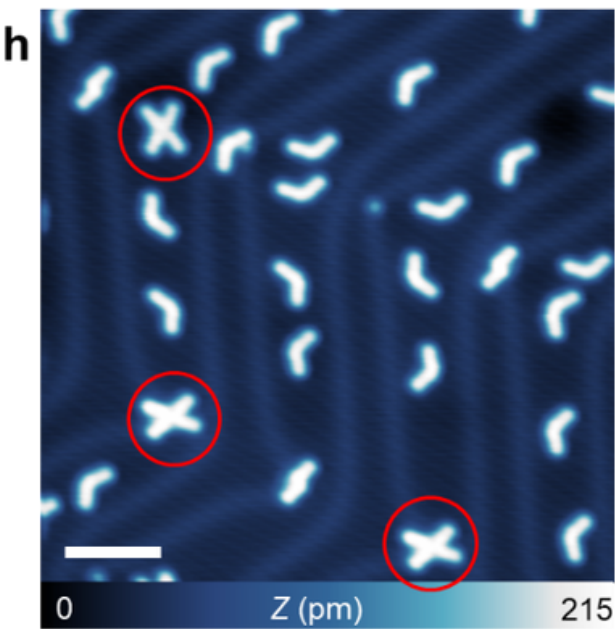

\section{Figure 5}

Probing the self-assembly steered intra- and intermolecular cyclization of precursor 1 via STM and AFM imaging. a, Schematic illustration of the debromination of precursor 1 leading to the formation of biradical intermediate 1', followed by the radical cyclization to form product 2 (left). The right path illustrates the Ullman coupling of 1' followed by the cyclodehydrogenation, yielding the products of 3 and 4, respectively. b, Large-scale STM image (metal tip, I = 100 pA, V = $1.5 \mathrm{~V}$ ) of the three products $(2,3$, and 4) after thermal annealing $(420 \mathrm{~K})$ of the dimer and trimer clusters of precursor 1 pre-assembled on $\mathrm{Au}(111)$. Scale bar is $5 \mathrm{~nm}$. c-e, ncAFM images of products 2, 3, and 4, respectively. Magnified ncAFM images (the bottom panels) highlight the molecular backbone of the center region (marked by dashed 
rectangle). Scale bars for c-e are $5 \AA$. f-h, Probe the distribution of reaction products at different precursor coverage ratio $(0.02,0.04$, and $0.09 \mathrm{ML}$ for $\mathrm{f}, \mathrm{g}$, and $\mathrm{h}$, respectively). Increasing the coverage yields a higher ratio of trimer clusters, therefore leading to more product 4 . Scale bars for $\mathrm{f}-\mathrm{h}$ are $5 \mathrm{~nm}$.

\section{Supplementary Files}

This is a list of supplementary files associated with this preprint. Click to download.

- JiongSI.docx

- JiongSI.docx 